\title{
Immunotherapy for Allergic Rhinitis
}

\section{Syed M Maseehur Rehman ${ }^{1,2 *}$ and Reginald Baugh}

${ }^{1}$ University of Toledo Medical Center, Toledo, Ohio, USA

${ }^{2}$ Medical Director, Asthma and Allergy Center Toledo, Ohio, USA

The article, "Prophylactic Inoculation against Hay Fever", the first ever research article promoting allergen immunotherapy was published in 1911 in Lancet. It was written by Leonard Noon, an investigator at the laboratory of the Department for Therapeutic Inoculation at St. Mary's Hospital, London, England [1]. Robert A. Cooke, who is considered the father of American allergy, began his investigation of pollen and sensitization in 1910, even before Noon's paper was published. His contribution, developing the basic method of immunotherapy as it is practiced today, was enormous. His treatment involved a build up phase of once a week injection of escalating dose of allergen during the first few months followed by one to twice a month maintenance treatment regimen over an optimal 4-5 years of treatment. Other modified regimes like cluster immunotherapy and rush immunotherapy were developed and are employed in certain patients to achieve desired therapeutic effect more rapidly.

Sub-cutaneous immunotherapy (SCIT) has been the mainstay of allergen immunotherapy for over a century. It is not only efficacious but also less expensive than pharmacotherapy. The greatest cost occurs in the first year and in the subsequent years it is much less expensive [2].

Even a century later immunotherapy is still the only disease modifying intervention that is currently available for the treatment of allergic rhinitis. Despite the considerable progress that has been made since then in our understanding of immunotherapy and the proof of its efficacy, this treatment is only used by $2-6 \%$ of appropriate patient candidates in the US. The reasons are multiple. SCIT is associated with inconvenience and discomfort from parental injection. The tough injection regimen discourages many to consider this as a treatment choice and many to fail to comply. SCIT also carries the risk of anaphylaxis, with fatalities.

First 100 years of immunotherapy was predominantly achieved by the sub-cutaneous route, but there were simultaneous efforts to look into oral immunotherapy (OIT) [3]. Oral immunotherapy dates back to as early as the 1900's when New York physician H.H. Curtis relieved his patients' hay fever by placing pollen antigen drops in their mouths. However, publication of the results had to wait until 1928 when $\mathrm{Dr}$ Black published the first ever data on OIT to pollens [4]. Fifty years passed until high dose OIT was found comparable to SCIT. The first double blind Placebo controlled DBPC study on SLIT was published in 1986 when a cross over trial demonstrated that oral Ragweed immunotherapy was an efficacious and safe alternative to SCIT [5]. In 1998 WHO endorsed the concept of SLIT and in the year 2000 the European Academy of Allergology and Clinical Immunology endorsed the use of OAT in Seasonal Allergic Rhinitis and Perennial Allergic Rhinitis and Allergic Asthma [2,6,7]. Several meta analyses as well as a Cochrane database review of multiple studies on SLIT, concluded that SLIT was effective and safe [8]. For patients with allergic rhinitis, oral therapy is very desirable but there are still questions about efficacy, safety, dose schedule and the use of multiple allergen therapy. A number of studies are under way in the US to address these questions.

The main goal of immunotherapy is to provide an effective, safe, preventive, economical and patient acceptable therapy. Current therapies have improved allergen tolerance but have failed to provide abso- lute desensitization (the conversion of a skin test result to negative). Further, there is need for interventions that can slow, stop or reverse disease progression utilizing an approach that is of short duration, safe and convenient for patients. Ideally, treatment that prevents onset of disease in genetically susceptible population will soon be possible.

\section{References}

1. Noon L. Prophylactic Inoculation against hay fever. Lancet 1922 111: 1572.

2. Hankin CS, Cox L, Lang D, Levin A, Gross G, et al. (2008) Allergy immunotherapy among Medicaid-enrolled children with allergic rhinitis: patterns of care, resource use, and costs. J Allergy Clin Immunol 121: 227-232.

3. Finegold I, Oppenheimer J (2010) Immunotherapy : the next 100 years. Ann Allergy Asthma Immunol 105: 394-398.

4. JH B (1928) The oral administration of pollens. J Lab Clinical Medicine 13: 709 .

5. Zuberbier T, Bachert C, Bousquet PJ, Passalacqua G, Walter Canonica G, et al. (2010) GA ${ }^{2}$ LEN/EAACI pocket guide for allergen-specific immunotherapy for allergic rhinitis and asthma. Allergy 65: 1525-1530.

6. JB (2006) Sublingual Immunotherapy: validated. Allergy 61: 5-6.

7. Bousquet J, Lockey R, Malling HJ (1998) Allergen immunotherapy: therapeutic vaccines for allergic diseases. A WHO position paper. J Allergy Clin Immuno 102: $558-562$.

8. Radulovic S, Caldorn M, Wilson D, Durham S (2011) Sublingual immunotherapy for allergic rhinitis (Review). Allergy 66: 740-752.

Corresponding author: Syed M Maseehur Rehman, Assistant Professor, University of Toledo Medical Center, Toledo, Ohio, USA, Tel: 419-408-2249; Fax: 419-843-8816; E-mail: smrallergy@yahoo.com

Received June 06, 2012; Accepted June 15, 2012; Published June 20, 2012

Citation: Rehman SMM, Baugh R (2012) Immunotherapy for Allergic Rhinitis. J Aller Ther S5:e001. doi:10.4172/2155-6121.S5-e001

Copyright: (C) 2012 Rehman SMM, et al. This is an open-access article distributed under the terms of the Creative Commons Attribution License, which permits unrestricted use, distribution, and reproduction in any medium, provided the original author and source are credited. 